\title{
Structure-Properties Relations in III-Nitride Nanostructures for Optoelectronics
}

\author{
R. C. Myers ${ }^{1,2,3}$, S. D. Carnevale ${ }^{1}$, A.T.M. G. Sarwar ${ }^{2}$, T. F. Kent ${ }^{1}$, P. J. Phillips ${ }^{4}$, C. Selcu ${ }^{3}$, R. F. Klie ${ }^{4}$, \\ F. Yang ${ }^{1}$, B. D. Esser ${ }^{1}$, D. W. McComb ${ }^{1}$ \\ 1. Dept. of Materials Science and Engineering, The Ohio State University, Columbus, Ohio, USA \\ 2. Dept. of Electrical and Computer Engineering, The Ohio State University, Columbus, Ohio, USA \\ 3. Dept. of Physics, The Ohio State University, Columbus, Ohio, USA \\ 4. Dept. of Physics, University of Illinois at Chicago, Chicago, Illinois, USA
}

III-Nitride based nanowire heterostructures are useful for optoelectronics applications across the visible and ultraviolet (UV) spectral range. A remarkable range of applications for these nanomaterials have been demonstrated, including solid-state-lighting, UV LEDs, lasers, photovoltaics, and photocatalysts. Compared with their thin film counterparts, III-N nanowires exhibit several key advantages including: inherently low defect densities (zero misfit dislocations and minimal stacking faults), lattice mismatch tolerance, and greater tunability of polarization and bandgap within a single heterostructure. In particular, single crystal III-N nanowires can be grown on a variety of substrates while retaining their high optical and electronic quality. Here we discuss a few illustrative examples of correlating the atomic scale structure determined by scanning transmission electron microscopy (STEM) measurements with the functional properties of the nanowire heterostructures.

Previously, we developed polarization-induced nanowire pn-junctions that take advantage of the built-in polarization dipole of the III-Nitride semiconductors [1]. To form these nanowire LEDs, p-GaN (Mgdoped) nanowires are first nucleated on $\mathrm{Si}$ (111) substrates using plasma-assisted molecular beam epitxay (MBE). Subsequently, a linearly compositionally graded AlGaN layer is grown using shutter pulsing, which leads to p-type conductivity. Next, a multiple InGaN quantum well (QW) active region is deposited, and finally a linearly graded AlGaN layers is grown leading to the top n-type section. High angle annular dark field (HAADF) STEM imaging and energy dispersive x-ray spectroscopy (EDXS) chemical composition mapping are used to characterize the composition gradients in order to quantify the polarization charge and determine the band edge diagram for the heterostructure. The directly acquired structural information thereby serves as a primary input to modeling the optoelectronic properties of the nanowires. Such measurements can be correlated with electronic measurements to quantitative predict the relation between free carrier concentrations and the polarization charge density [2].

However, STEM imaging of a handful of wires can lead to misleading interpretation of the entire ensemble due to inhomogeneity. In such cases, STEM techniques are complimented by lower resolution methods that can average over the sample inhomogeneity. As an example, we describe the determination of the nanowire polarity distribution using a combination of annular bright field (ABF) STEM imaging, which is sensitive to low $\mathrm{Z}$ elements, along with selective chemical etching of Ga-polar versus N-polar orientation of GaN in basic conditions (Fig. 1) [3,4]. By etching nanowires for long times, all N-polar nanowires can be removed from the substrate leaving any Ga-polar nanowires on the surface. ABFSTEM of the remaining wires confirms their Ga-polarity. In this way, SEM analysis of the pre- and post-etch surface allows determination of the dominant polarity and the quantitative distribution of polarity [5], which is confirmed by selective ABF-STEM imaging of the remaining (post-etch) nanowires. 
Finally, we describe use of STEM imaging in its more traditional role to determine the structural quality of a new type of self-assembled structure and to help explain the growth mechanism. The structures are called micro-mushrooms based on their cap and stem shape (Fig. 2). The stem consists of an InN nanowire. The cap consists of InN that grows laterally by adatom diffusion along the underside of the cap. This mechanism results in free standing microdisks of InN. This is a remarkable result for InN, which cannot be grown into large single crystals, but for which thin films always contain huge numbers of defects. The outer edge of the micro-mushroom caps exhibit a perfect wurtzite crystal structure free of extended defects. This is verified by performing plan-view STEM revealing a perfect honeycomb lattice. To our knowledge, such a honeycomb structure has never been previously observed for IIINitrides and is indicative of the lack of any stacking faults in the free-standing InN layers [6].

[1] S. D. Carnevale et al., Nano letters 12, 915 (2012).

[2] A. G. Sarwar et al., Appl. Phys. Lett. 106, 032102 (2015).

[3] K. Hestroffer et al., Physical Review B 84, 245302 (2011).

[4] L. Largeau, et al., Crystal Growth \& Design 12, 2724 (2012).

[5] S. D. Carnevale, et al., Nano letters 13, 3029 (2013).

[6] The authors acknowledge funding from the NSF CAREER award (DMR-1055164)

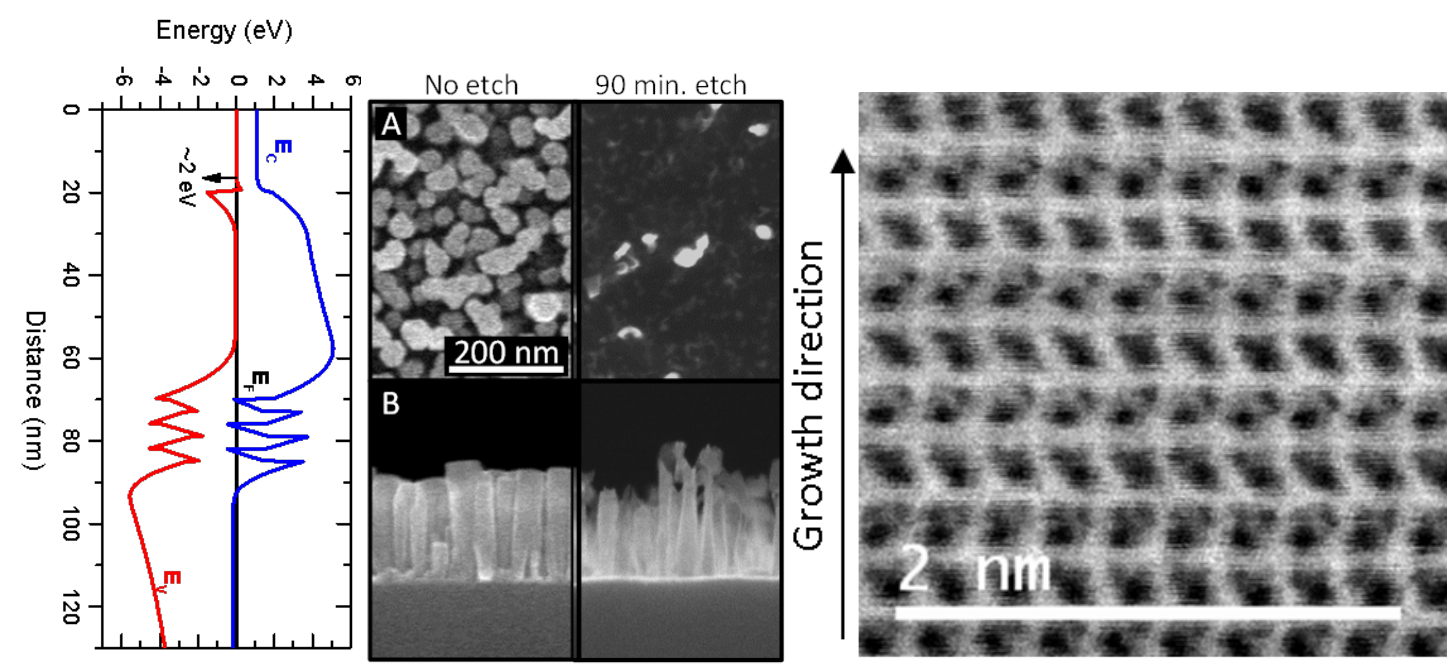

Figure 1. (left) Band edge diagram of a polarization-induced III-Nitride nanowire LED. (middle) Nanowire polarity-selective etching, and (right) direct ABF-STEM imaging of polarity.

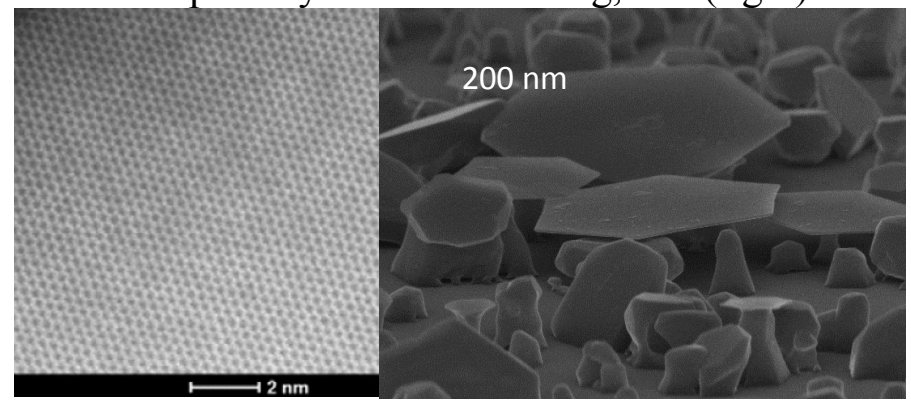

Figure 2. (left) Plan-view HAADF-STEM imaging through the cap of a self-assembled InN micromushroom. (right) Side-view SEM image of InN micromushrooms. 